\title{
"Gebe ich jetzt meine Daten preis oder nicht?““ Privatheit und Datenschutz in der Frühen Kindheit
}

\author{
Senta Pfaff-Rüdiger, Andreas Oberlinner, Susanne Eggert und Andrea Drexl
}

\begin{abstract}
Datenschutz und der verantwortungsbewusste Umgang mit eigenen und fremden Daten bei der Mediennutzung ist ein komplexes Thema, das besondere Herausforderungen mit sich bringt: Für viele Eltern ist es nicht einfach, an konkreten Beispielen zu erläutern, wie Daten verarbeitet und zu welchen Zwecken sie von Anbietern genutzt werden. Datenmissbrauch und die Bedeutung von Datenschutz und Privatheit sind darüber hinaus keine Phänomene, denen die Kinder in ihrer alltäglichen Mediennutzung häufig begegnen. Vor diesem Hintergrund ein Bewusstsein für die eher abstrakten Themen Privatheit und Datenschutz in der frühen Kindheit zu entwickeln und im medienerzieherischen Alltag umzusetzen, ist umso schwieriger. In einer qualitativen Langzeitstudie mit Familien mit Kindern im Alter von null bis sechs Jahren wurden die Haltungen und (Medienerziehungs-)Praktiken der Eltern im Umgang mit den Daten ihrer Kinder erhoben. Dabei konnten anhand der Kriterien Relevanz des Themas und Umsetzung im medienerzieherischen Handeln drei unterschiedliche Typen herausgearbeitet werden. Es wurde deutlich, dass die Auseinandersetzung und der Umgang mit dem Bereich Datenschutz und Privatheit von Kindern im Kleinkindalter insbesondere mit der medienerzieherischen Haltung der Eltern zusammenhängen.
\end{abstract}

\section{Einleitung}

Die Mediatisierung von Gesellschaft und Familie führt dazu, dass sämtliche Lebensbereiche mit digitalen Informations- und Kommunikationstechnologien durchdrungen werden. Kinder kommen dadurch immer früher mit digitalen Medien in Berührung und Eltern sehen sich mit der Aufgabe konfrontiert, ihre Kinder vom ersten Lebenstag an bei der Entwicklung eines kompetenten Medienumgangs zu begleiten und zu unterstüt- 
zen. Aufgrund der kognitiven kindlichen Entwicklung bedeutet dies zunächst, dass Eltern ihren Kindern eine souveräne Gestaltung des Alltags mit digitalen Medien vorleben und ihnen damit als Vorbild dienen, an dem die Kinder sich in ihrem eigenen Handeln orientieren können. Dies erfordert auf Seiten der Eltern die Reflexion und Bewusstheit des eigenen Medienhandelns und damit verknüpft eine Sensibilität für die medienbezogenen Bedürfnisse ihrer Kinder (vgl. Eggert/Wagner 2016). Da Kinder im frühkindlichen Stadium sich dieser Bedürfnisse zum Teil nur unzureichend oder (noch) gar nicht bewusst sind und diese (noch) nicht äußern können, sind die Eltern in der Verantwortung, an ihrer Stelle zu entscheiden. Mit Blick auf den Umgang mit Daten der Kinder heißt das auch, Entscheidungen zu treffen, deren mögliche Konsequenzen vielleicht erst in der Zukunft liegen. Dies gelingt den Eltern in unterschiedlichem Maße.

Hier setzt der Beitrag an. Im Zentrum stehen die Haltungen und das medienerzieherische Handeln von Eltern zum Thema Privatheit und Datenschutz in der frühen Kindheit, und damit bei Eltern von Kindern, die selbst noch kein Bewusstsein von Privatheit und Datenschutz haben. Welche Haltung haben Eltern zu Datenschutz, Privatheit und Sharenting und wie setzen sie dies in ihrem medienerzieherischen Handeln um? Im Rahmen der Langzeitstudie Familien-Medien-Monitoring wurden 2019 hierzu 17 Familien qualitativ befragt. Der Beitrag geht im Folgenden zunächst auf Privatheit und Datenschutz in der frühen Kindheit ein (Kapitel 2), bevor das methodische Vorgehen vorgestellt (Kapitel 3) und die zentralen Ergebnisse (Kapitel 4) diskutiert werden. Eine Typologie der unterschiedlichen medienerzieherischen Herangehensweisen an das Thema Privatheit macht abschließend (Kapitel 4.5) Einflussfaktoren erkennbar und gibt Hinweise darauf, wie Eltern in ihrer medienerzieherischen Kompetenz unterstützt werden können.

\section{Privatheit und Datenschutz in der frühen Kindheit}

Das Aufwachsen in einer sich kontinuierlich wandelnden Medienumwelt bringt Themen wie Datenschutz und Privatheit mit sich. Im Rahmen der Medienerziehung beschäftigen sich Eltern damit häufig erst, wenn es die eigenständige Nutzung der Kinder von Smartphone und Internet betrifft (vgl. Kutscher/Bouillon 2018). Im Fokus der wissenschaftlichen Auseinandersetzung standen dabei lange das Medienhandeln der Kinder und die Gefahren, denen Kinder durch Dritte online ausgesetzt sind. Ausgeblendet wurde dabei, welche Folgen das Medienhandeln der Eltern selbst für die Kinder haben kann (Kutscher 2019: 9f.). Im Folgenden geht es um den 
Umgang der Eltern mit Privatheit und Datenschutz in Familien mit Kindern im Alter von 0 bis 6 Jahren (frühe Kindheit) und damit um einen Bereich, der sehr stark vom Handeln der Eltern geprägt ist.

\subsection{Privatheit in der frühen Kindheit}

In der Annahme, dass Eltern ihre Kinder am besten kennen und einschätzen können, agieren Eltern von Kindern im Alter zwischen null und sechs Jahren als deren Treuhänder*innen und vertreten ihre Rechte (vgl. $\mathbb{1 0 4}$ BGB). Kinder verfügen in diesem Alter noch nicht über die kognitiven Fähigkeiten, um einen Wunsch nach Privatheit auszubilden und zu artikulieren (vgl. Stapf 2019: 18) oder über das Gefühl von Scham, ihr Bedürfnis nach Privatheit zum Ausdruck zu bringen (Walper/Maywald 2019: 3). Ebenso wird davon ausgegangen, dass Erwachsene aufgrund eines gewissen Erfahrungsvorsprunges und Informiertheit abwägen können, welche Folgen medienbezogenes Handeln mit sich bringen kann (vgl. Kutscher 2019: 9). Eltern sind sich jedoch oft der frühkindlichen Privatsphäre nicht bewusst (Stapf 2019: 18f.). Dadurch wird die Privatheit der Kinder, die Grundlage eines selbstbestimmten Lebens in der digitalen Welt ist, gefährdet, obwohl Kinder gleichzeitig ein Recht auf Privatheit (Art. 16 UN Kinderrechtskonvention) sowie ein Recht am eigenen Bild ( $\$ 22$ KunstUrhebG) haben.

Für Eltern sind Privatheit und Datenschutz wichtige Themen (DIVSI 2015: 17, Kutscher 2019: 12). Oftmals offenbaren sie aber persönliche Informationen über ihre Kinder im Netz (Naab 2019: 98). Das Privacy Paradox betrifft damit nicht nur die Preisgabe eigener Informationen, sondern auch die Preisgabe von persönlichen Informationen der Kinder. Grundsätzlich wird zwischen der informationellen, der psychologischen und der sozialen Privatheit unterschieden (vgl. Burgoon 1982). Informationelle Privatheit, d. h. Daten, die angegeben werden, um sich auf sozialen Netzwerken anzumelden, wird vor allem im Kontext einer aufkommenden datafizierten Kindheit thematisiert, bei der immer mehr Daten bereits von Kleinkindern online zu Verfügung stehen (Siibak/Traks 2019: 116), sei es durch Sharenting, die Nutzung von Kinderapps oder digitalem Spielzeug (Barassi 2018: 169f.). Die psychologische Privatheit bezieht sich auf meist intime Informationen, die geteilt werden, um die eigene Identität oder Beziehungen zu gestalten (Naab 2019: 98), während es bei der sozialen Privatheit darum geht, selbst bestimmen zu können, sich dem Kontakt zu anderen zu entziehen (Braun/Trepte 2017: 7). Eltern ist es dabei ein „wichtiges Anliegen, dass ihre Kinder verstehen, dass Inhalte - Fotos, Texte, persönli- 
che Daten etc. - die im Internet stehen, öffentlich sind" (Wagner et al. 2016: 23). Maßnahmen können dabei auf drei Privatheitsebenen umgesetzt werden: der kommerziellen, der institutionellen und der interpersonellen Privatheit (Livingstone et al. 2019). Die gewerbliche Datenverwendung durch Unternehmen kann als kommerzielle Privatheit beschrieben werden. Hier zeigt sich ein Unbehagen insbesondere bei personalisierter Werbung (Barassi 2018: 170). Die institutionelle Privatheit umfasst Daten, die im Kontext von Kindertageseinrichtungen, der Regierung oder von Gesundheitseinrichtungen gesammelt und ausgewertet werden. Beim Sharenting ist die interpersonelle Privatheit besonders wichtig. Im Sharenting „verbindet sich die seit langer Zeit zum Doing Family gehörende Praxis des Fotografierens von Familienmitgliedern (...) durch die Eltern mit der digitalen sozialen Netzwerkpraktik des Postens und Teilens" (Kutscher 2019: 8); es geht vorzugsweise um das Beziehungsmanagement, familialen Zusammenhalt und die Vermittlung von Kontinuität (Brosch 2018: 76, Schier 2013: 51). Hier liegt der Fokus auf der relationalen Ebene und der Frage, wie durch Privatheit Grenzen in Beziehungen ausgehandelt werden, beispielsweise welche Informationen wann mit wem geteilt werden (Livingstone et al. 2019: 11). Die Bedürfnisbefriedigung der Eltern steht dabei im Kontrast zur Datensparsamkeit und den Rechten der Kinder. Die Eltern müssen folglich abwägen, welche Daten sie wo preisgeben.

\subsection{Sharenting als Familienpraxis}

Durch die zunehmende Mediatisierung der Gesellschaft wird Familie immer häufiger über digitale Medien (re-)produziert und Sharenting hat sich als eine familiale Alltagspraxis etabliert (Autenrieth 2017: 147, Schlör 2019: 8). Das Phänomen Sharenting lässt sich nach vier Kategorien unterscheiden (Brosch 2018: 79). Zum einen nach (1) dem Kanal, über den Kinderbilder geteilt werden. Hiermit sind nicht nur unterschiedliche SocialMedia-Anwendungen oder Messenger gemeint, sondern auch, ob die Bilder als Profil- bzw. Statusbild genutzt oder im privaten Chat verschickt werden. Die Häufigkeit der Nutzung (2) und die Abwägung, mit welchem Adressat"innenkreis (3) die Bilder geteilt werden, stellen weitere Kategorien dar. Dabei geht es vor allem um das unsichtbare Publikum und die Tatsache, dass Bildmotive, die zu einem nicht zu vernachlässigenden Teil peinlich für die Kinder sind, unbeabsichtigt in eine breitere Öffentlichkeit gelangen können (Brosch 2016: 230f., Kutscher/Bouillon 2018: 7). Es ist nahezu unmöglich, das Teilen und Verschicken von Bildern auf den gewünschten Adressat*innenkreis zu begrenzen und eine weitere Verbrei- 
tung zu kontrollieren bzw. zu verhindern (vgl. z.B. UNICEF 2017: 92). Geteilt werden in Social Media häufig glückliche Momente des Familienlebens. Im Fokus stehen dabei inhaltlich (4) besondere Ereignisse oder Alltagsimpressionen. Oft sind Kinder bereits durch Ultraschallaufnahmen oder Bilder kurz nach der Geburt im Netz präsent (Autenrieth 2014: 102). Hier wird die Ambivalenz des Themas deutlich, da beim Sharenting eine Vielzahl von Kinderrechten verletzt werden können. Eine in den Medien häufig thematisierte Gefahr ist die Verbreitung von Kinderbildern in Pädophilen-Foren. Fotos, auf denen Kinder nackt oder nur teilweise bekleidet sind, werden durch Dritte von privaten Profilen heruntergeladen und zweckentfremdet. Problematisch sind auch Bilder, welche die Kinder in anderen Kontexten identifizierbar machen. Personenbezogene Daten wie der volle Name, die Adresse oder der aktuelle Aufenthaltsort können missbraucht werden.

\subsection{Privatheit und Datenschutz als Teil der elterlichen Medienerziehung}

Sharenting und der Umgang mit Datenschutz und der Privatheit der Kinder ist eingebettet in das medienerzieherische Handeln der Eltern. Studien zeigen, dass Eltern im Zuge der Medienerziehung ihren Kindern die Kompetenzen vermitteln wollen, ihre Privatsphäre zu schützen und die Konsequenzen des eigenen Handelns abzuschätzen (DIVSI 2015: 108, Wagner et al. 2016: 23). Für die kindliche Mediennutzung ist die Balance zwischen klaren Regeln, Vertrauen zwischen Eltern und Kindern und der Grad der Kontrolle durch die Eltern von Bedeutung. Gelingt dies, können Eltern ihrem Kind dabei helfen, ein Bewusstsein für seine Privatheit zu entwickeln und Medien und ihre Inhalte den eigenen Zielen und Bedürfnissen entsprechend kompetent zu nutzen (Walper/Maywald 2019: 3).

Die Studie „Kinder.Bilder.Rechte“ (2018) zeigt, dass Eltern Datenschutz und die Privatheit der Kinder zwar als wichtige Themen wahrnehmen, aber nicht über das nötige Wissen und die Fähigkeiten verfügen, um diese in der Praxis ausreichend zu schützen. Die Grundlage der elterlichen Datenschutzstrategien ist laut Kutscher „[e]ine Melange von Halbinformiertheit, Unsicherheit, Hilf- und Machtlosigkeit aber auch Gewöhnung an die Nutzungslogiken der digitalen Dienste“ (Kutscher 2019: 12). Vielfach werden dabei (un-)bewusst Kinderrechte verletzt, da der Wunsch nach eigener Bedürfnisbefriedigung stärker wirkt als der die Rechte der Kinder zu schützen (Naab 2019: 108f., Kutscher/Bouillon 2018: 8). Eingesetzte Strategien, um Privatheit und informationelle Selbstbestimmung zu gewährleisten, können dabei nicht getrennt werden von der Motivation, soziale Netzwer- 
ke oder andere Dienste zu nutzen (Brüggen/Wagner 2017: 138). Darüber hinaus haben die Eltern keine konsistente Vorstellung davon, wie sie Medienkompetenz und Medienwissen ihrer Kinder im Bereich Privatheit fördern können (Naab 2019: 98). Ihrer Verantwortungs- und Vorbildfunktion sind sich die Eltern kaum bewusst. Auch die Folgen ihrer Social-Media-Aktivitäten können sie nur begrenzt abschätzen (Kutscher/Bouillon 2018: 14). Je geringer die Kompetenz der Eltern im Umgang mit digitalen Medien, desto weniger Sicherheitsmaßnahmen ergreifen sie zum Schutz ihrer Kinder (DIVSI 2015: 133). Da die Eltern in der frühen Kindheit eine Treuhänder*innen-Rolle für ihre Kinder übernehmen, hat ihr Medien- und medienerzieherisches Handeln einen großen Einfluss darauf, welche Kompetenzen Kinder später in diesem Bereich erwerben können. Lange wurde bei der Frage nach Privatheit von Kindern ausgeblendet, welche Folgen das Medienhandeln der Eltern selbst für die Kinder haben kann. Die Studie fokussiert deshalb auf das medienerzieherische Handeln und die Haltung der Eltern zu Privatheit und Datenschutz in der frühen Kindheit und fragt auch danach, wie es gelingen kann, dass Eltern bereits in der frühen Kindheit, Privatheit und Datenschutz als wichtige medienerzieherische Themen erkennen und in ihr medienerzieherisches Handeln integrieren.

\section{Methodisches Vorgehen und Stichprobe}

Die Teilstudie „Familien-Medien-Monitoring“ (FaMeMo) fragt nach der Bedeutung digitaler und mobiler Medien in Familien mit Kindern im Alter von 0 bis 8 Jahren, wie sich Kinder diese Medien im Gesamtkontext der sie umgebenden Medienwelt aneignen und wie Eltern sie dabei begleiten. In einer Längsschnittstudie wurden 20 Familien bayernweit von 2017 bis 2020 in sechs Erhebungen mit qualitativen Leitfadeninterviews befragt. Neben der kontinuierlichen Erfassung der sich verändernden Mediennutzung und Begleitung durch die Eltern, wurden zusätzlich unterschiedliche Schwerpunktthemen gesetzt, um vertiefende Einblicke in bestimmte Bereiche zu erlangen. In der fünften Erhebung wurde mit gezielten Fragen auf die Praxis des Sharenting und auf Regeln bezüglich des Datenschutzes in der Familie eingegangen.

Die Auswahl der Familien erfolgte nach einer Quotenstichprobe (Akremi 2014: 273). Die Kriterien waren dabei das Geschlecht und Alter der Fokuskinder zu Beginn der Erhebung, die infrastrukturellen Rahmenbedingungen der Haushalte (städtische Kontexte, ländliche Räume) sowie die Bildung der Eltern, erhoben über die Ausbildungshintergründe der Eltern (niedriger/höher, vgl. Abb. 1). Eine Voraussetzung zur Teilnahme war, 
dass digitale Medien in der Familie vorhanden sind und auch genutzt werden. Die Medienaffinität der Eltern war kein Auswahlwahlkriterium, im Verlauf der Studie stellte sich aber heraus, dass die Familien digitale Medien unterschiedlich stark nutzen. Von den 20 Familien zu Beginn waren 2019 noch 17 Familien in der Befragung dabei, diese Panelmortalität wurde in der Zusammenstellung des Sample einkalkuliert (vgl. Stein 2014: 144).

Abbildung 1: Zusammensetzung des Samples nach Kriterien, Stand: 2019

\begin{tabular}{|c|c|c|c|c|c|c|}
\hline \multirow[t]{2}{*}{ Kriterium } & $\begin{array}{l}\text { Alter } \\
2017^{1}\end{array}$ & & & & \multirow[t]{2}{*}{ Start 2017} & \multirow[t]{2}{*}{ Stand 2019} \\
\hline & $1 \mathrm{Jahr}$ & 2 Jahre & 3 Jahre & 4-5 Jahre & & \\
\hline Anzahl der Fokuskinder & 6 & 3 & 6 & 5 & 20 & 17 \\
\hline $\begin{array}{l}\text { Geschlecht Fokuskind } \\
(\mathrm{m} / \mathrm{w})\end{array}$ & $2 / 4$ & $2 / 1$ & $3 / 3$ & $2 / 3$ & $9 / 11$ & $9 / 8$ \\
\hline $\begin{array}{l}\text { Infrastruktureller Bezugs- } \\
\text { raum }(\text { Stadt/Land })^{2}\end{array}$ & $4 / 2$ & $0 / 3$ & $3 / 3$ & $3 / 2$ & $10 / 10$ & $8 / 9$ \\
\hline $\begin{array}{l}\text { Bildungshintergrund } \\
\text { Eltern (höher/niedriger) }\end{array}$ & $3 / 3$ & $2 / 1$ & $2 / 4$ & $3 / 2$ & $10 / 10$ & $9 / 8$ \\
\hline
\end{tabular}

Die Leitfadeninterviews fanden meist bei den Familien zu Hause statt und dauerten 60 bis 90 Minuten. Die Interviews wurden vollständig transkribiert, anonymisiert und anschließend mit MAXQDA codiert. Die Auswertung fand dabei deduktiv theoriegeleitet und induktiv aus dem Material heraus statt (vgl. Meyen et al. 2019). Abschließend wurde eine Typologie entwickelt, die sich an der empirisch fundierten Typologiebildung nach Kluge (2000) orientiert. Nach der ersten Auswertung wurde deutlich, dass sich die Eltern vor allem darin unterscheiden,

1. welche persönliche Relevanz sie dem Thema Datenschutz und Privatheit zuschreiben,

2. ob sie Datenschutz und Privatheit auch in ihre medienerzieherische Praxis integrieren.

Anhand dieser zwei Kriterien wurden Gemeinsamkeiten und Unterschiede zwischen den Eltern deutlich, die zu einer Typenbildung sowie einer Charakterisierung und Namengebung führten. Um Einflussfaktoren ableiten zu können, wurden Kontextfaktoren in die Typologie einbezogen (vgl. Schorb/Theunert 2000).

1 Zum Zeitpunkt der fünften Erhebung, in deren Kontext Sharenting abgefragt wurde, waren die Kinder zwei Jahre älter.

2 Unter den Bezugsraum Land werden ländliche Räume und Kleinstädte gefasst, der Bezugsraum Stadt meint mittlere und große Städte. 


\section{Privatheit und Datenschutz im Familien-Medien-Monitoring}

\subsection{Zwischen smarten Geräten und eigenständiger Nutzung: Mediennutzung und Medienerziehung in den Familien}

Seit Beginn der Erhebungen sind in den Familien umfangreich digitale Mediengeräte vorhanden. So besitzen alle Eltern Smartphones. Laptops, Computer oder Tablets sind als zusätzliche Geräte neben den meist exklusiv persönlich genutzten Smartphones ebenfalls zu finden. Nur in den wenigsten Haushalten gibt es keine Fernsehgeräte, häufig verfügen die Familien über Smart-TVs oder sind mit einem Streaming-Stick ausgestattet. Die Familien ohne Fernsehgerät streamen Videos über Laptops oder Tablets. Daneben gibt es vereinzelt auch andere Geräte wie Smartwatches, 3D-Drucker, Konsolen sowie in drei Familien Sprachassistenzsysteme.

\section{Mediennutzung in den Familien: Zwischen Streaming und Sprachassis- tenzsystemen}

Die Mediennutzung der Eltern ist neben dem Fernsehen und der vereinzelten Nutzung des Laptops zum Arbeiten sehr stark auf das Smartphone konzentriert. Besonders WhatsApp, aber auch Facebook und vereinzelt Instagram werden intensiv für den Austausch mit Bekannten, Freund"innen und Verwandten genutzt. Daneben wird das Handy als Allrounder für Online Shopping, als Wecker, für Fotos oder zum Telefonieren verwendet. Ähnlich breit ist auch das Nutzungsverhalten der Kinder: Sie haben auf unterschiedliche Art und Weise Zugang zu digitalen Medien, die rezeptive Zuwendung dominiert dabei. So gibt es nur wenige Kinder, die nicht eine Fernsehsendung oder gestreamte Videos ansehen dürfen. YouTube spielt dabei in vielen Familien eine große Rolle und variiert von der gemeinsamen Nutzung auf dem Fernsehgerät bis zur alleinigen Nutzung des Kindes auf dem Smartphone der Eltern. Neben der rezeptiven Nutzung audiovisueller Medien nutzen die Kinder auch andere Anwendungen auf mobilen Geräten, zum Beispiel fotografieren sie mit dem Smartphone oder nutzen Sprachassistenzsysteme wie Alexa. So wird beispielsweise Alexa bei Familie Huber $^{3}$ zum Musikhören genutzt, während das Kind spielt. Adrian (3) versteht es dabei, Alexa selbst per Sprachsteuerung zu bedienen. Vereinzelt werden auch Medienangebote wie Skype oder WhatsApp genutzt, damit das Kind mit den Großeltern Kontakt halten kann. Auch sprachgesteuerte

3 Die Namen der Familien sind anonymisiert. 
Streaming-Sticks wie der Fire-TV-Stick von Amazon werden von einigen Kindern bereits selbst bedient. Mit zunehmendem Alter stehen den Kindern andere Medienangebote zu Verfügung. So durfte der Sohn von Familie Huber bereits mit zwei Jahren den Sprachassistenten bedienen, Zugang zu Fernsehinhalten bekam er aber erst nach und nach. Mit vier Jahren darf er nun auch selbstständig altersgerechte Spiele auf dem Tablet spielen. Auch bei vielen anderen Kindern hat sich im Untersuchungszeitraum die eigenständige Nutzung erhöht, die Kinder machen zum Teil Fotos mit den Smartphones ihrer Eltern oder nutzen bereits YouTube Kids alleine.

\section{Medienerziehung in den Familien: Regeln und Vorbildfunktion}

Die Mediennutzung läuft in vielen Familien sehr strukturiert ab. Oft gibt es klare Regeln, die die Nutzung von Geräten zeitlich und inhaltlich festlegen und in die Alltagsstruktur integrieren (vgl. Oberlinner et al. 2018). Ausnahmen gibt es dann, wenn Außergewöhnliches im Leben der Familien passiert, beispielsweise wenn ein Kind krank ist. Die Regeln werden aber nur selten den Kindern gegenüber begründet, sondern meist lediglich gesetzt. Die Eltern achten insgesamt auf altersgerechte Angebote und schließen bestimmte Inhalte aus, die sie als ungeeignet erachten. Viele Eltern möchten beim eigenen Umgang mit ihren Mediengeräten gute Vorbilder für ihre Kinder sein. Dies gelingt manchen nach eigener Einschätzung gut, andere sehen sich selbst als schlechte Vorbilder. Sie sorgen sich, dass die Kinder ihr Verhalten kopieren oder sie ihren Kindern zu wenig Beachtung schenken. Nur wenige Eltern benennen klare Regeln für sich selbst, wie zum Beispiel den Verzicht auf das Handy beim Essen.

\subsection{Zwischen Datensparsamkeit und personalisierter Werbung: Haltungen zu Privatheit und Datenschutz}

Privatheit und Datenschutz sind für alle befragten Eltern relevante Themen. Je nachdem, wie Eltern das Thema wahrnehmen, richten sie ihr medienerzieherisches Handeln danach aus (vgl. Eggert 2019). Die Haltungen sind dabei sehr unterschiedlich und reichen von einer „digital konservativen“, auf Datensparsamkeit bedachten Einstellung bei Familie Schäfer $(\mathrm{EH} 2 \text {, Sohn 3,5 Jahre })^{4}$ bis zu einer „ein stückweit vielleicht gutgläubig

4 Die Altersangaben zu denselben Kindern können im Text variieren, da die Belege sich immer auf die jeweilige Erhebungswelle (EH1 bis EH5) beziehen und jeweils angegeben wird, wie alt die Kinder zum Zeitpunkt dieser Erhebung waren. 
oder naiv[en]" Haltung bei Frau Walter, die sich nicht vorstellen kann, wer sich derart für ihren „private[n] Kram“ interessieren könnte, „um das jetzt zu missbrauchen“ (EH5, Tochter, 5,5 Jahre). Herr Schäfer führt seine Haltung auf sein Informatikstudium „zu Beginn des Internets“ zurück, in dessen Kontext das Thema Datenschutz selbstverständlich gewesen sei (EH4, Sohn 4,5 Jahre).

Die Haltung der Familien lässt sich nach der je betroffenen Privatheitsperspektive differenzieren. Die psychologische Privatheit wird - auch über Sharenting hinaus - deutlich häufiger angesprochen als die informationelle Privatheit, die eher im Kontext von kommerzieller und institutioneller Privatheit thematisiert wird. ${ }^{5}$ Betrachtet man die informationelle Privatheit, dann lässt sich festhalten, dass die meisten Eltern über wenig Wissen verfügen, was mit den eigenen Daten passiert. Ausnahmen sind Familien Ziegler, Unger und Witt. Sie thematisieren beispielsweise die Möglichkeit, sich über die Kamera des Tablets zuzuschalten und so Daten sammeln zu können, um über Bilder auf den Aufenthaltsort der Kinder schließen zu können, aber auch, dass „Perverse“ Zugang zu Kinderbildern bekommen könnten (Frau Witt, EH3, Sohn, 3,5 Jahre). Über weiterführendes Wissen über Datengenerierungs- und -verarbeitungsprozesse verfügt - abgesehen von Herrn Schäfer - von den befragten Eltern niemand.

Sehr wohl gibt es aber bei vielen ein Bewusstsein darüber, dass für kommerzielle Interessen Daten gesammelt werden. Es geht um personalisierte Werbung oder die Tatsache, dass Daten preisgegeben werden müssen, um online einkaufen zu können. Frau Ritter ist sich dessen bewusst, aber auch skeptisch, ob sie „es hinkriegen kann, das so zu verschleiern, dass die [Firmen] nichts davon mitkriegen" (EH5, Sohn, 3 Jahre). Die Alltagsbelastungen führen eher dazu, dass die Datenfreigabe bewusst in Kauf genommen wird. Eine Familie ist darüber hinaus bereit, die eigenen Kinder zu Werbezwecken auf die Webseite der eigenen Arbeitsstätte zu stellen (Frau Baumer, EH5, Sohn, 5,5 Jahre). Dass über WhatsApp Metadaten an den Facebook-Konzern weitergegeben werden, wird von den Eltern kaum thematisiert. Lediglich Familie Schäfer (Signal) und Herr Ritter (Threema) haben sich für einen alternativen Messenger entschieden.

Auch die institutionelle Privatheit spielt für das Familienleben eine Rolle, wenn es um Krippen, Kindergärten oder bei älteren Geschwistern um die

5 Auf Selbstoffenbarung (self-disclosure) und psychologische Privatheit wird im Kapitel über Sharenting noch ausführlich eingegangen werden. An dieser Stelle soll nur festgehalten werden, dass hier eine große Anzahl an Eltern zumindest ein Unbehagen darüber ausdrückt, dass Bilder von den Kindern online kursieren. 
Schule geht. Während Frau Brandt sich über die Facebook-Seite der Krippe ihres Sohnes freut und lobend erwähnt, dass die Gesichter der Kinder dort nicht zu sehen seien, fühlen sich andere Familien eher in die Einverständniserklärung für die Verwendung der Daten gezwungen. So führt Herr Walter beispielsweise an, dass, ,jetzt auch immer alles unterschrieben werden" müsste (EH5, Tochter, 5,5 Jahre) und Frau Lindmüller möchte ihrer älteren Tochter keine Nachteile durch das Verweigern der Unterschrift bescheren (EH5, Tochter, 4,5 Jahre).

\subsection{Zwischen Beziehungsarbeit und Shaming: Sharenting}

Im Bereich der interpersonellen Privatheit spielt vor allem das Sharenting eine Rolle, das für viele Familien bereits zum „Gewohnheitsding“ (Frau Flacher, EH5, Sohn, 4 Jahre) geworden ist, das nicht mehr hinterfragt wird. Was den Adressat"innenkreis und den Kanal angeht, unterscheiden die Eltern, ähnlich wie in der Studie „Kinder.Bilder.Rechte“ (2018), zwischen Facebook und WhatsApp. Fast alle Familien lehnen ein Posten von Bildern auf Facebook ab. Sie wollen die Kinder „lieber ins Album bringen“ als sie auf Facebook für alle „ausstellen“ (Frau Beckmann, EH5, Sohn, 6,5 Jahre). Auch hier steht die Sorge im Vordergrund, nicht kontrollieren zu können, wer das Bild „mal für was verwendet“ (Herr Ziegler, EH4, Tochter, 5 Jahre) und die Kinder später nicht beschämen zu wollen.

Im Gegensatz dazu werden in fast allen Familien Bilder auf WhatsApp geteilt. Die Familien unterscheiden hier zwischen den einzelnen Verbreitungsmöglichkeiten. Nur wenige stellen Bilder der Kinder auch in den Status oder nutzen sie als Profilbild. In Bezug auf den Status nahmen zwei Familien kulturelle Unterschiede wahr: Frau Grün und Frau Lindmüller mussten den amerikanischen Cousin bzw. die mexikanische Bekannte darauf hinweisen, doch bitte keine Bilder ihrer Kinder in den eigenen Status zu stellen. Die Familien betonen, dass sie auf WhatsApp nur Bilder mit „ganz vertrauenswürdigen Personen“ (Herr Bogner, EH5, Tochter, 6,5 Jahre) teilen. Meist handelt es sich bei den Adressat"innen um enge Verwandte oder Freund"innen. Die Eltern gehen dabei davon aus, dass die Bilder nicht weitergegeben werden. Viele sind sich allerdings darüber nicht sicher und vermuten eher, dass „die Bilder nach kurzer Zeit wieder gelöscht werden" (Frau Färber, EH5, Tochter, 3 Jahre) oder die Großeltern nicht über die notwendige Medienkompetenz verfügen, um sie weiterzugeben. Das Bedürfnis, die Großeltern an der eigenen Lebenswelt teilhaben zu lassen, ist jedoch wichtiger als die Kontrolle über Daten. Besonders gilt dies für herausragende Familienereignisse wie eine Geburt oder den Erfolg ei- 
nes Enkelkindes beim Sport. Die Häufigkeit variiert dabei von „sehr dezentem" Austausch von Bildern (Frau Beckmann, EH5, Sohn, 6,5 Jahre) bis zum täglichen Update bei Familie Ritter oder Walter.

Was den Inhalt der geteilten Bilder angeht, sind sich die Familien einig, dass Badebilder, die die Kinder (halb-)nackt zeigen, unangebracht sind. Wegen eines Bilds auf einem Bagger seien die Kinder dagegen ,in zehn oder auch 20 Jahren nicht böse" (Frau Baumer, EH5, Sohn, 5,5 Jahre). Die Eltern wägen also ab, welche Bilder sie mit anderen teilen, sie beziehen aber die Kinder nicht in ihre Entscheidung mit ein und verletzen so deren Recht am eigenen Bild. Wie andere Studien zeigen, haben Kinder mit zunehmendem Alter aber ein Gefühl dafür, welche Bildinhalte für sie in Ordnung sind und möchten hier auch gefragt werden (Kutscher/Bouillon 2018, jugendschutz.net 2019). Gerade in der frühen Kindheit ist es wichtig, dass die Eltern ihr Elternprivileg hier nicht leichtfertig auslegen.

\subsection{Klare Vorbilder sind gefragt: Medienerzieherisches Handeln zum Thema Datenschutz}

Viele der befragten Eltern sehen bei ihren Kindern (noch) keinen Handlungsbedarf (vgl. Kutscher/Bouillon 2018) und thematisieren Datenschutz und Privatheit eher im Kontext der Smartphonenutzung von verwandten oder befreundeten Jugendlichen. Vereinzelt überlegen die Eltern, wie sie später mit der Privatsphäre ihrer Kinder umgehen wollen. Dass ihre Kinder bereits eigenständig Internetangebote nutzen und so Daten weitergeben, wenn sie beispielsweise YouTube nutzen, mit der Oma auf WhatsApp videotelefonieren oder Sprachassistenzsysteme benutzen, ist kaum einem Elternteil bewusst. In den Familien mit Sprachassistenzsystemen sorgen sich die Mütter eher darum, dass die Kinder hier einen Befehlston lernen (Frau Baumer, EH1, Sohn, 3,5 Jahre) bzw. sprechen in einem Atemzug von Datenschutz, während sie Alexa darum bitten, die Musik leiser zu stellen (Frau Huber, EH5, Sohn, 3,5 Jahre).

Regeln werden den Kleinkindern in Bezug auf Datenschutz und Privatheit kommunikativ (noch) nicht vermittelt und beziehen sich stattdessen eher auf das elterliche Medienhandeln, beispielsweise darauf, keine Kinderbilder online zu posten. In einigen Familien gilt die eingeschränkte Regel, nur „unbedenkbare [Bilder] von hinten“ (Herr Bogner, EH5, Tochter, 6,5 Jahre) online zu stellen. Einige Eltern handeln aus, welche Inhalte von den Kindern online gestellt werden können. Dass die Eltern mit ihrem Medienhandeln im Bereich Datenschutz und Privatheit den Kindern als Vorbild dienen, ist nur den wenigsten bewusst, ebenso wenig ihre Treu- 
händer*innenschaft (vgl. Naab 2019). Dies mag auch daran liegen, dass die Kinder das Handeln der Eltern mit Bezug auf Privatheitsfragen - anders als einen achtsamen Umgang mit den Geräten - schlechter wahrnehmen können und die Vorbildrolle deshalb kommunikativ hergestellt werden müsste.

Privatheit und Datenschutz sind zudem innerhalb der Medienerziehung nur ein Thema unter vielen und im Familien-Medien-Monitoring deutlich weniger präsent als in quantitativen Befragungen zur Medienerziehung (vgl. z.B. DIVSI 2015) oder zu elterlichem Jugendmedienschutz (Brüggen et al. 2017). Für die Mehrheit der Familien haben sie nicht den gleichen Stellenwert wie beispielsweise Fragen nach einer altersgerechten Nutzung oder nach Medienwirkungen. Nur Frau Unger und Frau Schäfer formulieren Privatheit und Datenschutz explizit als Erziehungsziel („da muss man halt aufpassen, was man macht oder was man postet, was man öffentlich macht", Frau Unger, EH5, Tochter, 4 Jahre). Bei den anderen Familien dominiert der Wunsch, ein "gesundes Mittelmaß“ zu finden (Frau Grün, EH5, Tochter, 3 Jahre), den Umgang mit Technik zu lernen bzw. den Kindern das notwendige Wissen zu vermitteln, um Selbstoptimierungsansprüche bzw. Informationen einordnen und bewerten zu können.

Die Eltern sorgen sich zum Teil um das Wohl ihrer Kinder, wenn sie über Shaming oder die Tatsache reflektieren, dass „das Internet [nichts] vergisst" (Frau Witt, EH3, Sohn, 3,5 Jahre), haben aber nicht die Rechte der Kinder im Blick. Diese werden nicht thematisiert. Frau Durr erwähnt stattdessen explizit, dass die Kinder in der frühen Kindheit noch nicht über die kognitiven und emotionalen Fähigkeiten verfügen würden, um die Folgen der Weitergabe von Daten verarbeiten zu können (EH3, Tochter, 3,5 Jahre).

\subsection{Typologie: Relevanz des Themas Privatheit in der Erziehung}

Basierend auf der Relevanz des Themas Privatheit und dem medienerzieherischen Handeln zum Datenschutz wurde eine Typologie gebildet, um herauszuarbeiten, wovon es abhängt, ob die Eltern über Datenschutz nachdenken und dies sogar in ihr medienerzieherisches Handeln integrieren (vgl. zum methodischen Vorgehen Kapitel 3). Typologisierungskriterien waren dabei die Relevanz, die Eltern dem Thema Datenschutz und Privatheit (hoch vs. niedrig) zuschreiben und ob sie die Relevanz des Themas auch in eigene medienerzieherische Maßnahmen umsetzen (ja vs. nein). Aus dem Material ließen sich drei Typen ableiten (vgl. Abb. 2). 
Abbildung 2: Typologie Privatheit in der Medienerziehung

\begin{tabular}{|l|c|c|}
\hline Relevanz/Umsetzung & $\begin{array}{l}\text { Umsetzung } \\
\text { in medienerzieherisches } \\
\text { Handeln }\end{array}$ & $\begin{array}{l}\text { keine Umsetzung } \\
\text { in medienerzieherisches } \\
\text { Handeln }\end{array}$ \\
\hline $\begin{array}{l}\text { Datenschutz wird als } \\
\text { relevantes Thema erkannt }\end{array}$ & Die Konsequenten & Die Pragmatischen \\
\hline $\begin{array}{l}\text { Datenschutz wird nicht als } \\
\text { relevantes Thema erkannt }\end{array}$ & Im Sample nicht vertreten & Die Sorglosen \\
\hline
\end{tabular}

\section{Typ 1: Die Konsequenten}

Neun Familien reflektieren ihr eigenes Medienhandeln und beziehen auch Privatheitsaspekte mit ein. Diese Familien verfügen über Wissen, was informationelle Privatheit angeht. Kinderbilder werden zwar auf Messengern mit der (Groß-)Familie geteilt, dabei wird aber stark nach Kontexten differenziert (wem wird welches Bild geschickt) und darauf geachtet, die Kinder nicht zu kompromittieren. Die Eltern unterscheiden dabei sowohl den Kanal als auch die Inhalte und die Häufigkeiten des Sharentings, teilen Fotos eher selten und nur bei besonderen Anlässen. Darüber hinaus fällt auf, dass das Thema Datenschutz und Privatheit in diesen Familien mit den Großeltern, den älteren Geschwistern oder zwischen den Eltern kommunikativ ausgehandelt wird. So hat Frau Unger bereits mit den älteren Geschwistern über die Folgen von öffentlichen Bildern gesprochen und Frau Ziegler mit ihrem Mann sowie Herr Bogner mit der Großmutter ausgehandelt, dass keine Bilder der Kinder auf Facebook gepostet werden. Die Eltern sehen sich zum Teil dabei auch als Vorbilder für die Kinder. Einen Sonderfall stellt Familie Schäfer dar, die über sehr explizites Wissen über Datenschutz verfügt und auch gesellschaftliche Folgen mitdenkt und diskutiert.

Die Mütter dieses Typs arbeiten häufig in sozialen Berufen, die Väter kommen zum Teil aus der Informatik und der Pädagogik. Die Kinder sind tendenziell schon älter und haben häufig ältere Geschwister. Anders als bei den anderen beiden Typen nutzen die Kinder bereits häufiger eigenständig Apps wie YouTube auf mobilen Geräten. Smarte Geräte wie Smartwatches oder Smart-TV sind eher selten. Was die Vorbildfunktion betrifft, machen sich die Eltern vor allem Gedanken um den „Aufmerksamkeitsschlucker Smartphone " (Herr Bogner, EH1, Tochter, 4 Jahre), sehen ihre eigene Mediennutzung kritisch und legen auf alternative Tätigkeiten der Kinder Wert. 


\section{Typ 2: Die Pragmatischen}

Diese fünf Eltern haben ein Problembewusstsein, was Datenschutz und Privatheit angeht und erkennen, dass sie Daten angeben müssen, wenn sie online aktiv sein wollen. Sie ergreifen aber nicht die entsprechenden Maßnahmen, um ihre Daten zu schützen bzw. verfügen nicht über die technischen Kenntnisse. Andere Bedürfnisse stehen im Vordergrund. Dies betrifft zum einen - auf der interpersonellen Privatheitsebene - das Verbundensein mit anderen Familienmitgliedern. Auf der kommerziellen Ebene geht es darum, sich den Alltag zu vereinfachen, indem Anfragen über Ale$x a$ gestellt werden oder beim Online-Shopping bewusst in Kauf genommen wird, dass die eigenen Daten dort gespeichert werden. Anders als bei den Sorglosen (vgl. Typ 3), findet bei den Pragmatischen Kommunikation über das Thema Datenschutz und Privatheit nur reaktiv statt, wenn beispielsweise Großeltern sich zu viele Rechte herausnehmen oder man sich an die Praxis der anderen Familienmitglieder anpasst. Frau Ritter überlegt zwar im Interview immer wieder, ob sie anders handeln könnte. Ihr fehlt aber - ähnlich wie den anderen Mitgliedern dieses Typs - eine Idee für Alternativen und damit ein Handlungswissen, das über reine Privatsphäreeinstellungen hinausgeht. $\mathrm{Zu}$ diesem Typ gehören eher Familien mit jüngeren Kindern (zu Beginn des Panels ein Jahr alt), die Eltern sind höher gebildet, die Mütter arbeiten alle, zum Teil auch in Vollzeit, darunter eine Journalistin und eine Lehrerin. Die Medienausstattung ist sehr umfangreich und es gibt einige Haushalte, in denen die Väter sich gerne mit den neuesten Geräten ausstatten. Auffällig ist, dass die Kinder in ihrer Mediennutzung sehr viel Musik hören, häufig Musikvideos auf YouTube sehen und mit den Großeltern zum Teil schon videotelefonieren. Anders als bei den Konsequenten, erleben die befragten Mütter ihre Vorbildrolle positiv und beziehen diese vor allem auf einen bewussten und reduzierten Umgang mit dem Smartphone.

\section{Typ 3: Die Sorglosen}

Drei Familien kennen das Thema Datenschutz und Privatheit, es ist aber für sie nicht handlungsrelevant - weder für das eigene Medienhandeln noch für die Medienerziehung. Darüber hinaus verfügen sie über wenig privatheitsbezogenes Wissen. Frau Walter kann als Reisekauffrau die "deutsche Angst vor Online-Buchungen“ nicht nachvollziehen, sie habe „immer gute Erfahrungen gemacht“ (Frau Walter, EH4). Insbesondere was die kommerzielle Privatheit angeht, gehen diese Eltern bewusst Risiken ein, stellen unter anderem das eigene Kind auf die berufliche Webseite zu Werbezwecken und missachten so die Kinderrechte. Diese Familien stehen 
viel mit anderen in Kontakt und teilen regelmäßig Fotos der Kinder online. Die Kinder sind in diesem Typ alle älter (zu Beginn drei Jahre alt) und haben meist ältere Geschwister. Die Mütter arbeiten und haben in ihren Berufen online mit Daten oder Werbung zu tun. Die Medienausstattung unterscheidet sich in den Familien nicht explizit von anderen. Die Kinder dürfen bereits mit älteren Geschwistern oder Nachbarn an der Konsole spielen. In ihrer eigenen Vorbildrolle sind diese Eltern unsicher und können sie weniger als die anderen Typen an einem konkreten (eigenen) Medienverhalten festmachen.

\section{Einflussfaktoren auf das Privatheitsverhalten}

Was beeinflusst nun aber, ob die Familien eher konsequent, pragmatisch oder sorglos im Umgang mit den Daten sind?

- Medienensemble zuhause: Hier fällt auf, dass die Pragmatischen sehr an Technologie interessiert sind und sich gerne mit den neuesten Geräten ausstatten. In diesem Typ finden sich deshalb auch deutlich mehr Smartgeräte als beispielweise bei den Konsequenten.

- Beruf: Unter den Konsequenten finden sich einige Informatiker, ein Pädagoge, der bereits beim Film gearbeitet hat und eine Rechtsanwaltsgehilfin. Sie haben berufsbedingt einen anderen Blick auf Datenschutz als die Sorglosen, die ebenso wie die Pragmatischen eine Faszination für Technik mitbringen, aber über Datenentstehungs- und -verwertungsprozesse nicht Bescheid wissen.

- Alter der Kinder: Unter den Pragmatischen sind nur Kinder, die zu Beginn ein oder zwei Jahre alt waren und noch wenig eigenständig digitale Medien nutzen, dafür aber bereits mit den Großeltern über Videotelefonie in Kontakt sind. Hier stellt sich auch die Frage, ob die Pragmatischen nicht auch aufgrund der Alltagsbelastung mit sehr kleinen Kindern Datenschutzfragen zurückstellen (müssen). Werden die Kinder älter und weitet sich ihr Nutzungsspektrum aus, reagieren die Eltern entweder mit Vorsicht (die Konsequenten) oder machen sich (weiterhin) keine Gedanken über Datenschutz und Privatheit (die Sorglosen).

- Ziele der Medienerziehung: Die Konsequenten sind die einzigen, die zumindest zum Teil Datenschutz und Privatheit als Ziel ihrer Medienerziehung definieren. Sie setzen es dabei neben aktive Gestaltungsmöglichkeiten mit den Medien. Da sie hier das selbstständige Handeln der Kinder in den Vordergrund rücken, werden Aspekte, die sie dabei schützen, wichtiger. Anders dagegen die Pragmatischen: Sie kritisieren vor allem den Konsum- und Selbstoptimierungsaspekt der Medien und wollen die Kinder darauf vorbereiten. Interessanterweise nehmen sie 
aber in Kauf, dass beim Konsum eigene Daten weitergeben werden. Die Sorglosen haben dagegen noch keine Ziele, wenn es um die Medienerziehung geht und verschieben das Thema auf das Schulalter. Hier lässt sich deutlich sehen, wie wichtig es ist, das eigene medienerzieherische Handeln auf Basis der damit verbundenen Ziele zu reflektieren. Wer Ziele benennen kann, kann diese auch ins eigene Handeln integrieren.

\section{Fazit}

Datenschutz und Privatheit sind Themen, die Eltern von Kindern im Alter von null bis sechs Jahren nicht fremd sind. Sie wünschen sich, dass die Daten, die sie im Rahmen ihrer Mediennutzung von sich selbst oder von ihren Kindern preisgeben, sicher sind und nicht missbraucht werden. Wie sie selber dazu beitragen können, welche technischen Sicherheitsvorkehrungen sie vornehmen können und welche Maßnahmen in ihrem alltäglichen Medienhandeln wie auch im Rahmen ihrer Medienerziehung zielführend sind, ist vielen Eltern nicht bewusst. Den Schutz ihrer eigenen und der Daten ihrer Kinder überlassen viele Eltern anderen und zeigen dabei zum Teil eine fatalistische und resignative Haltung. Zum Ziel ihrer Medienerziehung machen Eltern den sicheren und verantwortungsbewussten Umgang mit Daten - eigenen und fremden - dann, wenn sie das Thema selbst als Herausforderung sehen und es sogar Einzug in ihre normativen Vorstellungen von Medienerziehung gefunden hat (die Konsequenten). Voraussetzung dafür ist, dass sie über ein ausführliches Medienstrukturwissen verfügen bzw. sich - beispielsweise ausgelöst durch Medienberichterstattung oder andere Personen - bereits mit dem Thema auseinandergesetzt haben. Dabei geht es weniger um die Mediennutzung der Kinder, sondern stärker um die elterliche Wahrnehmung von Medienerziehung und die eigenen Ansprüche (vgl. Eggert 2019: 111). Mit Blick auf eine Stärkung der elterlichen Medienerziehungskompetenz gilt es vor diesem Hintergrund, Eltern die Bedeutung, die sie für ihre Kinder als Vorbilder sowie als wichtigste Ansprechpersonen haben, ins Bewusstsein zu rufen. 


\section{Literatur}

Akremi, Leila (2014): Stichprobenziehung in der qualitativen Sozialforschung. In: Baur, Nina / Blasius, Jörg (Hg.): Handbuch Methoden der empirischen Sozialforschung. Wiesbaden: VS Verlag, S. 265-282.

Autenrieth, Ulla (2014): Die ,Digital Natives' präsentieren ibre Kinder - Eine Analyse der zunehmenden (Selbst-) Visualisierung von Familie und Kindheit in Onlineumgebungen. In: Studies in Communication Sciences 10 (35), S. 99-107.

Barassi, Veronica (2018): The child as datafied citizen. Critical questions on data justice in family life. In: Mascheroni, Gianna / Ponte, Cristina / Jorge, Ana (Hg.): Digital parenting. The challenges for families in the digital ages. Göteborg: Nordicom, S. 93-102.

Braun, Max / Trepte, Sabine (2017): Privatheit und informationelle Selbstbestimmung: Trendmonitor zu den Einstellungen, Meinungen und Perspektiven der Deutschen. Stuttgart: Universität Hohenheim.

Brosch, Anna (2016): When the child is born into the Internet: Sharenting as a growing trend among parents on Facebook. In: The New Educational Review 43 (1), S. 225-234.

Brosch, Anna (2018): Sharenting - why do parents violate their children's privacy? In: The New Educational Review 54 (4), S. 75-85.

Brüggen, Niels / Dreyer, Stephan / Drosselmeier, Marius / Gebel, Christa / Hasebrink, Uwe / Rechlitz, Marcel (2017): Jugendmedienschutzindex: Der Umgang mit onlinebezogenen Risiken - Ergebnisse der Befragung für Eltern und Heranwachsenden. Herausgegeben von FSM - Freiwillige Selbstkontrolle Multimedia-Diensteanbieter e.V. Online verfügbar unter: www.fsm.de/jugendmedienschutzindex (Abfrage am: 16.04.2020).

Brüggen, Niels / Wagner, Ulrike (2017): Recht oder Verhandlungssache? Herausforderungen für die informationelle Selbstbestimmung aus Perspektive der Jugendlichen. In: Friedewald, Michael / Lamla, Jörn / Roßnagel, Alexander (Hg.): Informationelle Selbstbestimmung im digitalen Wandel (DuD-Fachbeiträge), Wiesbaden: VS Verlag, S. 131-146.

Burgoon, Judee K. (1982): Privacy and Communication. In: Communication Yearbook 6 (1), S. 206-249.

Deutsches Institut für Vertrauen und Sicherheit im Internet (DIVSI) (2015): DIVSI U9-Studie Kinder in der digitalen Welt. Eine Grundlagenstudie des SINUS-Instituts Heidelberg im Auftrag des Deutschen Instituts für Vertrauen und Sicherheit im Internet (DIVSI). Online verfügbar unter: https://www.divsi.de/wp-content/uploads/2 015/06/U9-Studie-DIVSI-web.pdf (Abfrage am: 24.3.2019).

Eggert, Susanne / Wagner, Ulrike (2016): Grundlagen zur Medienerziehung in der Familie. Expertise im Rabmen der Studie MoFam - Mobile Medien in der Familie. Online verfügbar unter: www.jff.de/studie_mofam (Abfrage am: 7.05.2020). 
Eggert, Susanne (2019): Familiäre Medienerziehung in der Welt digitaler Medien: Ansprüche, Handlungsmuster und Unterstützungsbedarf von Eltern. In: Fleischer, Sandra / Hajok, Daniel (Hg.): Medienerziehung in der digitalen Welt. Grundlagen und Konzepte für Familie, Kita, Schule und Soziale Arbeit. Stuttgart: Kohlhammer, S. 105-118.

jugendschutz.net (2019): Report. Kinderbilder auf Instagram. Wann werden Persönlichkeitsrechte von Kindern verletzt? Online verfügbar unter: https://www.servicestelle -jugendschutz.de/wp-content/uploads/sites/17/2019/10/Report_Kinderbilder_auf _Instagram.pdf (Abfrage am: 16.4.2020).

Kluge, Susanne (2000): Empirically Grounded Construction of Types and Typologies in Qualitative Social Research. Forum Qualitative Research 1 (1). Online verfügbar unter: http://www.qualitative-research.net/index.php/fqs/article/view/1124/2500 (Abfrage am: 16.04.2020).

Kutscher, Nadia (2019): Kinder. Bilder. Rechte. Wie Kinderrechte in der digitalen Welt durch die Eltern alltäglich und ungewollt beeinträchtigt werden. In: Frühe Kindheit 22 (2), S. 6-13.

Kutscher, Nadia / Bouillon, Ramona (2018): Kinder. Bilder. Rechte. Persönlichkeitsrechte von Kindern im Kontext der digitalen Mediennutzung in der Familie. Berlin: Schriftenreihe des Deutschen Kinderhilfswerks Heft 4.

Livingstone, Sonia / Stoilova, Mariya / Nandagiri, Rishita (Hg.) (2019): Children's data and privacy online. Growing up in a digital age. An evidence review. Online verfügbar unter: http://www.lse.ac.uk/my-privacy-uk/Assets/Documents/Childre ns-data-and-privacy-online-report-for-web.pdf (Abfrage am: 09.11.2019).

Meyen, Michael / Löblich, Maria / Pfaff-Rüdiger, Senta / Riesmeyer, Claudia (2019): Qualitative Forschung in der Kommunikationswissenschaft. Eine praxisorientierte Einführung (2). Wiesbaden: Springer VS.

Naab, Thorsten (2019): Parents' online self-disclosure and parental social media trusteeship. How parents manage the digital identity of their children. In: Medien Pädagogik Zeitschrift für Theorie und Praxis der Medienbildung (35), S. 97-115. Online verfügbar unter: https://www.medienpaed.com/article/view/656 (Abfrage am: 24.03.2020).

Oberlinner, Andreas / Eggert, Susanne / Schubert, Gisela / Jochim, Valerie / Brüggen, Niels (2018): Medienrituale und ihre Bedeutung für Kinder und Eltern. Erster Bericht der Teilstudie „Mobile Medien und Internet im Kindesalter - Fokus Familie“. München: JFF - Institut für Medienpädagogik in Forschung und Praxis. Online verfügbar unter: www.jff.de/mofam (Abfrage am: 16.04.2020).

Schier, Michaela (2013): Räumliche Entgrenzungen - Multilokales Familienleben. In: Wagner, Ulrike (Hg.): Familienleben: Entgrenzt und vernetzt?! München: Kopaed Verlag, S. 39-55.

Schlör, Katrin (2019): Doing Family mit Medien. Impulse für eine lebenslange medienpädagogische Familienbildung. In: Akademie der Diözese Rottenburg- Stuttgart (Hg.). Im Dialog. Beiträge aus der Akademie der Diözese Rottenburg-Stuttgart. Aufwachsen mit Medien - Mediensozialisation und -kritik heute (41. Stuttgarter Tage der Medienpädagogik) o.Jg. (1), S. 7-22. 
Schorb, Bernd / Theunert, Helga (2000): Kontextuelles Verstehen der Medienaneignung. In: Paus-Hasebrink, Ingrid / Schorb, Bernd (Hg.): Qualitative Kinder- und Jugendforschung. Theorien und Methoden: Ein Arbeitsbuch. München: Kopead. Verlag, S. 33-57.

Siibak, Andra / Traks, Keily (2019): The dark sides of sharenting. In: Catalan Journal of Communication \& Cultural Studies 11 (1), S. 115-121.

Stapf, Ingrid (2019): „Ich sehe was, was du auch siehst“. Wie wir die Privatsphäre der Kinder im Netz neu denken sollten und was Kinder möglicherweise dabei stärkt - ein kinderrechtlicher Impuls. In: Frühe Kindheit 22 (2), S. 14-23.

Stein, Petra (2014): Forschungsdesigns für die quantitative Sozialforschung. In: Baur, Nina / Blasius, Jörg (Hg.): Handbuch Methoden der empirischen Sozialforschung. Wiesbaden: Springer VS, S. 135-151.

UNICEF (2017): The State of The World's Children: Children in a Digital World 2017. Online verfügbar unter: https://www.unicef.org/publications/files/SOWC _2017_ENG_WEB.pdf (Abfrage am: 10.04.2020).

Wagner, Ulrike / Eggert, Susanne / Schubert, Gisela (2016): MoFam - Mobile Medien in der Familie. Langfassung der Studie. Online verfügbar unter: https://www.jff. de/fileadmin/user_upload/jff/projekte/mofam/JFF_MoFam1_gesamtStudie.pdf (Abfrage am: 10.04.2020).

Walper, Sabine / Maywald, Jörg (2019): Editorial. In: Frühe Kindheit 22 (2), S. 3. 